\title{
Corrigendum: CORO1C is Associated with Poor Prognosis and Promotes Metastasis Through PI3K/AKT Pathway in Colorectal Cancer
}

\begin{abstract}
Zongxia Wang ${ }^{1}$, Lizhou Jia ${ }^{1,2}$, Yushu sun ${ }^{3}$, Chunli Li $^{1}$, Lingli Zhang ${ }^{4}$, Xiangcheng Wang ${ }^{5,6 *}$ and Hao Chen ${ }^{2,7 *}$

${ }^{1}$ Cancer Center, Bayannur Hospital, Bayannur, China, ${ }^{2}$ Department of Pathology, Wannan Medical College, Wuhu, China, ${ }^{3}$ Department of Oncology, Inner Mongolia Autonomous Region Cancer Hospital, Hohhot, China, ${ }^{4}$ Department of Ophthalmology, Inner Mongolia Autonomous Region People's Hospital, Hohhot, China, ${ }^{5}$ Department of Nuclear Medicine, The Affiliated Hospital of Inner Mongolia Medical University, Hohhot, China, ${ }^{6}$ Key Laboratory of Inner Mongolia Autonomous Region Molecular Imaging, Inner Mongolia Medical University, Hohhot, China, ${ }^{7}$ Faculty of Medical Science, Jinan University, Guangzhou, China
\end{abstract}

Keywords: CORO1C, colorectal cancer, prognosis, metastasis, Akt

\section{A corrigendum on}

OPEN ACCESS

Edited by:

Haishi Qiao,

China Pharmaceutical University,

China

Reviewed by:

Ern Yu Tan,

Tan Tock Seng Hospital, Singapore

${ }^{*}$ Correspondence: Xiangcheng Wang

guyan@nmgfy.com

Hao Chen

ha0chen@wnmc.edu.cn

Specialty section:

This article was submitted to Molecular Diagnostics and

Therapeutics,

a section of the journal

Frontiers in Molecular Biosciences

Received: 18 June 2021

Accepted: 14 July 2021

Published: 30 August 2021

Citation:

Wang Z, Jia L, Yushu sun, Li C, Zhang L, Wang $X$ and Chen $H$ (2021)

Corrigendum: CORO1C is Associated with Poor Prognosis and Promotes Metastasis Through PI3K/AKT

Pathway in Colorectal Cancer.

Front. Mol. Biosci. 8:727347.

doi: 10.3389/fmolb.2021.727347
CORO1C is Associated with Poor Prognosis and Promotes Metastasis through PI3K/AKT Pathway in Colorectal Cancer

by Wang, Z., Jia, L., Sun, Y., Li, C., Zhang, L., Wang, X., Chen, H. (2021). Front. Mol. Biosci. 8:682594. doi: $10.3389 /$ fmolb.2021.682594

In the original article, there was a mistake in the caption for Figure $\mathbf{4 A}$ as published. The incorrect caption stated NCM460 cell lines are normal gastric epithelial cells, however, NCM460 cell lines are normal colorectal epithelial cells. The correct caption for Figure 4 appears below. Additionally, there was a mistake in Figure $\mathbf{1}$ as published. Figure $1 \mathrm{C}$ is immunofluorescence and Figure 1D is immunocomplex by Co-IP. OE-ControlTrop2 should appear on the top row and OE-Trop2 should appear on the bottom row in Figure 1C. Representative images for these parts contain small issues which have been corrected. The corrected Figure 1 appears below.

FIGURE 4. The effects of CORO1C knockdown on CRC growth and metastasis in vitro and in vivo. (A) Levels of CORO1C1 protein expression in CRC cell lines and normal colorectal epithelial cells (NCM460) determined by western blotting. (B) COCA2 and HCT116 cells showed a significant decrease in protein level after shCORO1C transfection. (C) CORO1C downregulation significantly inhibited the proliferation of both cell lines. (D) A significant decrease in cell anchorage-dependent growth was detected after CORO1C knockdown. (E, F) Decreased CORO1C expression impaired abilities of migration (E) and invasion (F) of CRC cells (scale bar, $150 \mu \mathrm{m}$ ). All quantitative data of in vitro assays were generated from three replicates $(\mathbf{G})$. The effects of CORO1C downregulation on the tumor growth in the xenograft mouse model $(n=6$ mice/per group). ${ }^{\star} p<0.05,{ }^{* *} p<0.01,{ }^{* * *} p<0.001$.

The authors apologize for this error and state that this does not change the scientific conclusions of the article in any way. The original article has been updated.

Publisher's Note: All claims expressed in this article are solely those of the authors and do not necessarily represent those of their affiliated organizations, or those of the publisher, the editors and the reviewers. Any product that may 

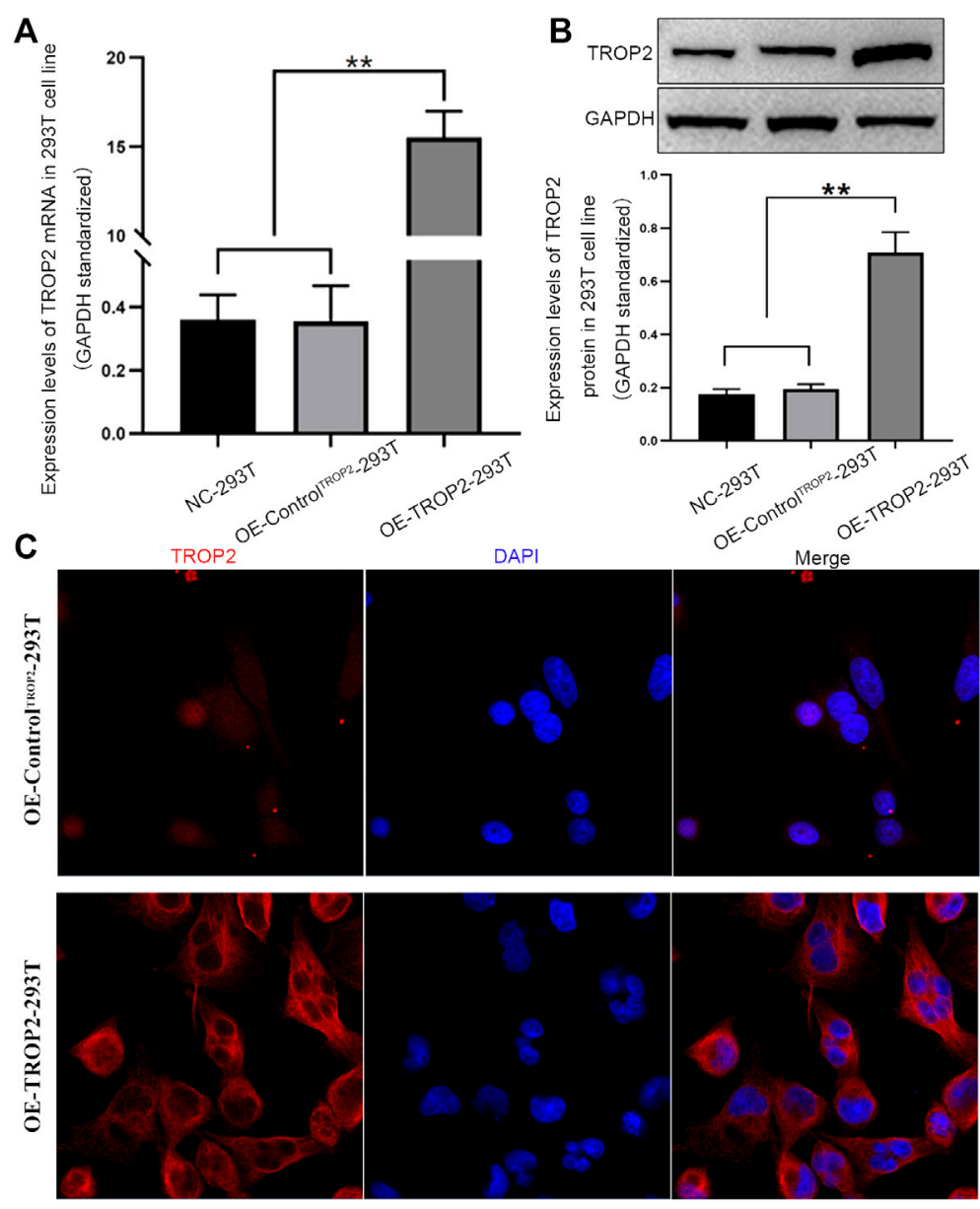

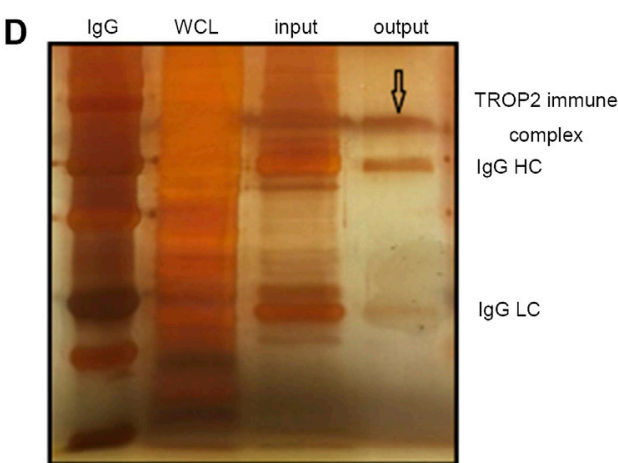

$\mathbf{E}$

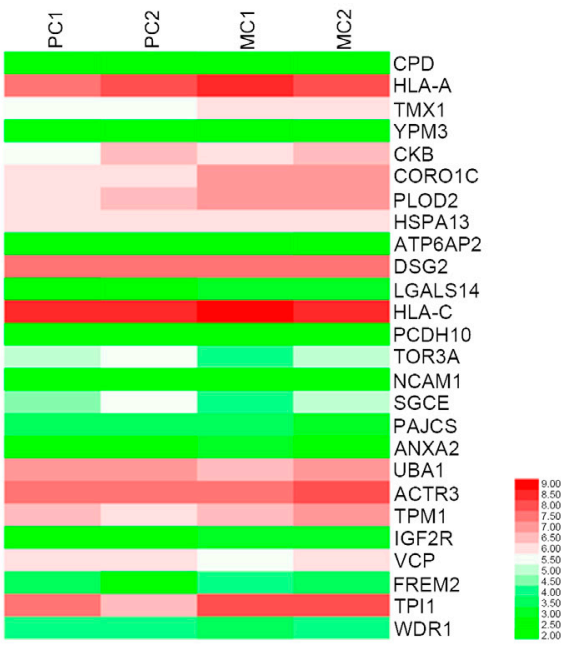

FIGURE 1 | Associations between CORO1C, TROP2, and CRC metastasis. Overexpression of Trop2 in 293 T cells was confirmed by (A) qRT-PCR, (B) Western blotting, and (C) Immunofluorescence. (D) Trop2 immunocomplex by Co-IP; (E) Heatmap of proteins interacting with Trop2 in PC and MC. The RNA-seq data were obtained from GSE28702. Red and green colors represent high and low gene expression, respectively. PC: primary CRC; MC: metastatic CRC, ${ }^{\star \star} p<0.01$.

be evaluated in this article, or claim that may be made by its manufacturer, is not guaranteed or endorsed by the publisher.

Copyright (c) 2021 Wang, Jia, Yushu sun, Li, Zhang, Wang and Chen. This is an open-access article distributed under the terms of the Creative Commons Attribution
License (CC BY). The use, distribution or reproduction in other forums is permitted, provided the original author(s) and the copyright owner(s) are credited and that the original publication in this journal is cited, in accordance with accepted academic practice. No use, distribution or reproduction is permitted which does not comply with these terms. 